\title{
How an Upper Secondary School Teacher Provides Resources for the Transition to University: A Case Study
}

\author{
José María Gavilán-Izquierdo ${ }^{1} \mathbb{C}$, Inés Gallego-Sánchez ${ }^{1^{*}} \mathbb{C}$
}

\author{
${ }^{1}$ Universidad de Sevilla, SPAIN \\ *Corresponding Author: inesgal@us.es
}

Citation: Gavilán-Izquierdo, J. M., \& Gallego-Sánchez, I. (2021). How an Upper Secondary School Teacher Provides Resources for the Transition to University: A Case Study. International Electronic Journal of Mathematics Education, 16(2), em0634. https://doi.org/10.29333/iejme/10892

\begin{tabular}{|c|c|}
\hline ARTICLE INFO & ABSTRACT \\
\hline Received: 10 Nov. 2020 & This paper analyzes the discursive activity of an upper secondary school teacher on the topic of the derivative. \\
\hline Accepted: 6 Feb. 2021 & $\begin{array}{l}\text { Specifically, we focus on the discursive activity when introducing the three essential concepts related to this topic: } \\
\text { the average rate of change, the derivative at a point, and the derivative function. The theoretical framework used } \\
\text { is the theory of commognition (Sfard, 2008). Using this framework, we identify different types of visual mediators } \\
\text { (symbols, graphs, gestures) and routines (mainly construction and substantiation ones, with various subtypes). } \\
\text { The analysis of these visual mediators and routines allows us to examine some resources students will have in } \\
\text { their transition to university discourse. We also draw some other conclusions and present implications for } \\
\text { teaching in this stage. }\end{array}$ \\
\hline
\end{tabular}

Keywords: teacher's discursive activity, commognition, routines, visual mediators, transition, derivative

\section{INTRODUCTION}

Research at university levels is a booming field and there are various domains of interest, including the transition to university (Biza et al., 2016). Different researchers have analyzed this transition (Clark \& Lovric, 2009; Corriveau \& Bednarz, 2016; Gueudet, 2008). For Clark and Lovric (2009), this transition (which they call a "rite of passage") has three phases, and two of them take place in upper secondary education (16-18 years). Numerous authors have found students' difficulties in this transition, especially in mathematics (De Guzmán et al., 1998; Gueudet, 2008; Panero et al., 2016; Rach \& Heinze, 2017; Rach \& Ufer, 2020; Rooch et al., 2016; Schüler-Meyer, 2018; Thoma \& Nardi, 2018).

One of the fundamental mathematical concepts in that transition is the derivative (Thomas et al., 2015), which appears in the curriculum in non-compulsory secondary education courses (16-18 years). There are several current studies on the teaching and learning of this concept, both at the secondary and university levels. It is important to highlight that the derivative is articulated through two concepts: the derivative of a function at a point and the derivative function, together with the relationship between them. Research in mathematics education has identified that a critical issue for understanding is the relationship between these two concepts (Badillo et al., 2011; García et al., 2012; Panero et al., 2016; Park, 2015; Zandieh, 2000). Park (2015), in the first year of university, and Panero et al. (2016), in the last years of secondary education, studied teachers' explanations in the transition process between the concepts of derivative at a point and derivative function (which the last call "genericization"). Park (2015) pointed out that this transition is frequently done simply by swapping the literal symbol for a variable, rather than focusing on how the function changes over an interval. According to Panero et al. (2016), spoken language, graphics, written language, symbols, and gestures must be consistent with the nature of the concept (at one point, local, or global) to promote students' understanding.

Furthermore, the derivative is a concept that has several contexts and layers: physical, graphic, slope, ratio, limit, function, etc. (Zandieh, 2000) and different forms of representation: symbolic, graphic, real situations, etc. (Behr et al., 1983). Various authors study the relationship between modes of representation of tasks and their understanding, or between the use of the concept image and its definition (Borji et al., 2018; Borji \& Sánchez, 2019; Dahl, 2017; Fuentealba et al., 2019; Pinto-Rojas \& Parraguez, 2017). Fuentealba et al. (2019) identified three levels of understanding of the derivative scheme (intra-inter-trans), which are based, among other aspects, on different modes of representation. Moreover, Borji and Sánchez (2019) concluded that the predominant mode of representation in secondary education is algebraic. For this reason, first-year undergraduate students may have difficulties in solving problems in other modes of representation or finding relations between them. Specifically, PintoRojas and Parraguez (2017) studied the problems of students in understanding the concept of derivative of a function at a point. They concluded that the graphic meaning presents more difficulties than the analytical one, which is often the most used. It is also 
important to note that the derivative concept has several applications in other areas. The most common is in physics, where the derivative is used to find the instantaneous speed, i.e., the instantaneous variation of position with respect to time (Zandieh, 2000).

Definitions and their construction constitute an important issue in upper secondary education. Lakatos (1976) considers the practice of defining as fundamental in the construction of mathematical conjectures and proofs, and Freudhental (1973) considers that it has the same relevance as proving. Other authors also highlight the importance of this practice and of definitions (de Villiers, 1998; Tall, 1991; Tall \& Vinner, 1981). In particular, Tall (1991) points out that defining and definitions are a characteristic of "the move from elementary to advanced mathematical thinking" (p. 20). Likewise, different authors have studied different types of definitions (Bayda \& Sutliff, 2020; Viirman, 2014) or errors in their use, such as Edwards and Ward (2008).

According to Morgan (2005), the way definitions are presented in school can affect the transition to advanced mathematics. Definitions at school are usually introduced through examples or through formalizing an existing concept, which is more typical of definitions in social sciences. Freudhental (1973) named this class of definitions as descriptive, as opposed to constructive (stipulative) ones. Similarly, Edwards and Ward (2008) state that some first-year undergraduate students have difficulties in understanding the difference between types of definitions, i.e., between "extracted" (common language) or "stipulative" (mathematical) definitions (Landau, 2001). Thus, while common language definitions inform the use of objects, mathematical definitions create concepts to be used and are free of connotations. Therefore, they conclude that mathematics teachers should address the special nature of definitions at any level, as well as their use and creation. According to Burn (2002), mathematics teachers at universities often use stipulative definitions and follow the definition-theorem-proof pattern. Still, students and educators criticize that pattern, since they argue that it does not help the student to learn the concepts and it does not represent the natural way of doing mathematics. More generally, Dreher et al. (2018) affirm that mathematics at university has a rigorous axiomatic-deductive structure, a higher level of abstraction, and symbolic language. By contrast, school mathematics is intuitive, inductive, and based on familiar, concrete things.

In recent years, sociocultural perspectives in mathematics education research (Lerman, 2001; Planas \& Civil, 2010) have highlighted the consideration of learning as a social and cultural construction. One of the most widely used sociocultural perspectives is the theory of commognition (Sfard, 2007, 2008). The term commognition derives from the union of the words communication and cognition. This theory considers mathematical discourse as the object of study and thinking as a type of intrapersonal discourse. In addition, it offers a holistic perspective of the processes and situations of teaching and learning in mathematics. In this theory, learning is seen as a process of progressive participation in more specialized discourses and can be initiated by imitation (Sfard, 2008). Specifically, teaching is defined as a communicative activity whose purpose is to bring students' discourse closer to a canonical discourse (Tabach \& Nachlieli, 2016). In this framework, the "teacher or instructor, is a person who assumes the role of the leading participant in the discourse while the student is a person who assumes the position of the follower in the discourse" (Tabach \& Nachlieli, 2016, p. 303). Concerning the transition stage, according to Thoma and Nardi (2018), a more explicit and systematic presentation of the differences between the discourses of secondary and university education is needed to facilitate the flexible movement between them. Likewise, Sfard (2014) indicates that university mathematical discourse has characteristics that differentiate it from school discourse.

Several researchers have recently used commognition to characterize teachers' and students' discursive activity (EmreAkdoğan et al., 2018; Güçler, 2013; Martín-Molina et al., 2020; Park, 2011, 2015; Tasara, 2018; Viirman, 2014). They conclude that the differences between discourses frequently cause difficulties in the teaching and learning processes. Furthermore, SchülerMeyer (2018) concludes that investigating students' practices in terms of their discourse in secondary education and the realizations they use is useful to understand the resources they have in their transition to university discourse.

Following Schüler-Meyer (2018), we consider the importance of the last years of the non-compulsory secondary education (1618 years) in the transition to university, and we characterize the teacher's mathematical discursive activity that emerges during the introduction of the derivative concept. As a theoretical framework, we use the theory of commognition (Sfard, 2007, 2008), looking for specific features in the teacher's discourse that help us understand what resources students may have in their transition to tertiary education.

In what follows, the basic principles of the theoretical background used in the study are introduced. Next, the methodology and the results of the analysis are presented, and, finally, the discussion and conclusions are shown.

\section{THEORETICAL FRAMEWORK}

We use in our analysis the theory of commognition (Sfard, 2007, 2008). This is a sociocultural theory that studies discourse and looks for changes in it, since learning is seen as "changing a discourse in a lasting way" (Sfard, 2008, p. 299). Thus, Sfard (2007, 2008) proposes four properties to characterize mathematical discourse: word use, visual mediators, narratives, and routines. These discursive properties are described below:

- Word use. It includes the use of mathematical words (for example, asymptote) and the use of common language words with mathematical meaning (for example, function or limit).

- Visual mediators. They are "providers of the images through which speakers identify the object of their speech and coordinate their communication" (Sfard, 2008, p. 147). Examples of visual mediators are tables, symbols, graphs, or manipulative materials.

- Narratives. "A narrative is any sequence of sentences framed as a description of objects, relationships between objects or processes with objects, which is subject to approval or rejection" (Sfard, 2008, p. 134). If they are considered true by 
the discourse participants (discursants), they are called "endorsed narratives". An example could be the statement "the derivative of a function at a point is the slope of the tangent line at this point".

- Routines. "They are characteristic repetitive patterns of a given discourse" (Sfard, 2008, p. 134). They do not usually appear explicitly but can be inferred from discourse by looking at the other properties. Examples of math routines are defining, testing, executing an algorithm, etc. According to Sfard (2008), the how and when of each routine must be considered. The how of a routine is "a set of metarules that determine, or just constrain, the course of the patterned discursive performance" (p. 208), and the when of a routine "is a collection of metarules that determine, or just constrain, those situations in which the discursant would deem this performance as appropriate" (p. 208).

Sfard (2008) declares that mathematical learning occurs when there are changes in the discourse, particularly in some of its properties: word use, visual mediators, narratives, or routines. According to Sfard, this learning is achieved through a progressive adjustment of the individual discourse to the leading discourse. Moreover, she states that the discursants need to be unanimous about which they consider the leading discourse and, that in a traditional classroom, it is usually the teacher's.

Routines are classified into three types: explorations, deeds, and rituals (Sfard, 2008). Thus, an exploratory routine (exploration) "is a routine whose performance counts as completed when an endorsable narrative is produced or substantiated" (Sfard, 2008, p. 224). An example of an exploration is finding the value of a limit.

Moreover, explorations can be divided into three types:

construction, which is a discursive process resulting in new endorsable narratives; substantiation, the action that helps mathematists decide whether to endorse previously constructed narratives; and recall, the process one performs to be able to summon a narrative that was endorsed in the past. (Sfard, 2008, p. 225)

Viirman (2014) classified the exploratory routines of construction and substantiation in a study on the teaching of the concept of function by university lecturers. His classification of these types of routines will be used in the data analysis.

Specifically, Viirman (2014) identifies several types of construction routines: construction of definitions by stipulation, exemplar, contrast or naming, construction of examples and counterexamples, algorithms, etc. Definitions that are constructed by exemplar, contrast, or naming can be considered descriptive (Freudhental, 1973); that is, they are based on pre-existing objects. Furthermore, the substantiation routines identified by Viirman (2014) include definition verification, that is, checking if an example satisfies a specific definition; other substantiation routines are proof and claim contradiction. He also includes examples of substantiation algorithms. Viirman (2014) does not propose a classification for recall routines, because they do not appear in his study. However, in our work, they appear and will be analyzed.

This article focuses on identifying and classifying exploratory routines and visual mediators in the upper secondary school teacher's discursive activity when teaching the derivative concept. The focus on routines is justified because, as Sfard (2008) states, "the search for discursive patterns is the essence of commognitive research" (p. 200), and we can consider "learning as a process of routinization" (Lavie et al., 2019, p. 153). Only exploratory routines are analyzed in this work because the focus is on teachers' discourse, which mainly has an explorative nature when introducing mathematical theory (Lavie et al., 2019). Specifically, how narratives are constructed and substantiated at this level will be examined. Visual mediators are analyzed due to the richness of the derivative concept in modes of representation and contexts of use (Zandieh, 2000). Furthermore, according to Sfard (2009), "gestures are crucial for the effectiveness of mathematical communication" (p. 197) and, as Alibali and Nathan (2012) indicate, "a better scientific understanding of gesture is crucial to forge a deeper understanding of educational communication and change in knowledge" (p. 249). Coinciding with Park (2015), we consider gestures as visual mediators in this work and classify them according to McNeill (1992). For this last author, the most important types of gestures are iconic, which visually resemble concrete referents; metaphorical, where "the pictorial content presents an abstract idea instead of a concrete object or event" (McNeill, 1992, p. 14); beats, which are repetitive gestures that "index the word or phrase that they accompany as significant" (p. 15), and deixis, a "pointing movement that selects a part of the gestural space" (p. 80).

Therefore, our research questions are: what are the exploratory routines and visual mediators present in the mathematical discursive activity of upper secondary school teachers when they explain the derivative concept? What do the characteristics of this discursive activity tell us about the resources that secondary school teachers are providing to their students in order to prepare them for their transition to university?

\section{METHODOLOGY}

In this section, we describe the participants and the context of our study, how the data have been collected and how the analysis has been carried out.

\section{Participants and Context}

We have employed a qualitative-interpretative methodology that takes the form of a case study (Yin, 2003). The participant in this research is a teacher, Anna (pseudonym), who is a graduate in mathematics with more than 20 years of teaching experience in secondary and upper secondary education. She was selected due to her willingness to collaborate with the researchers and her potential to learn from her participation in this study. Anna taught in a secondary school of a medium-sized city (approximately 130,000 inhabitants). The class in which we collected our data had 23 students in their first year of upper secondary education (1618 years), which is non-compulsory. The students had not received prior instruction on the subject of the derivative, so this was 


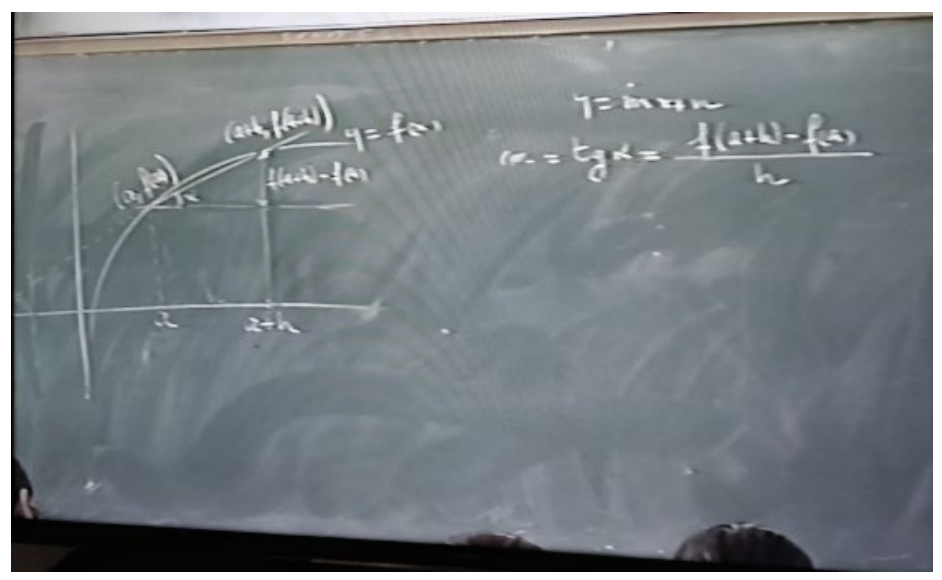

Figure 1. Slope of the Secant Line

their first contact with this mathematical concept. The unit devoted to teaching the derivative occupied 11 classes of 50 minutes on average. The researchers did not intervene at any time in the planning and development of those classes. In each of those, Anna gave a master class on the board, interspersing theoretical explanations with examples and textbook exercises.

\section{Data Collection}

Our research data are the audio and video recordings of three class sessions dedicated to the topic of the derivative, in which Anna introduced the main aspects. The recordings have been transcribed verbatim, with indications of what was being done at each moment (writing on the board, gestures, etc.), which appear in square brackets, and were supported by screenshots. In the transcripts, what Anna says is preceded by the letter A, and what the students say by the letter S. We do not distinguish which specific student or students speak at each moment because we focus mainly on the teacher's discourse. Nevertheless, we include some students' interventions because it helps the reader to get an idea of how the conversation developed.

\section{Analysis Procedure}

Focusing on the teacher's mathematical discursive activity, we identified its characteristic properties: word use, visual mediators, narratives, and inferred from them the routines. Data were analyzed individually by each researcher, then their findings were compared and, in the few cases in which there were discrepancies, other researchers were consulted. Specifically, in this work, we have focused on the characterization and classification of visual mediators and of exploratory routines. In the following, we show an example of how these two properties were identified in the discourse.

Anna (A): We are going to take a point " $a$ " and we are going to give it an increment ... we are going to put " $a+h$ " here ... "( $a, f$ $(a))$ " okay, and this point? " $(a+h, f(a+h))$ "...so that the ordinates are " $f(a)$ " and " $f(a+h)$ " and the abscissas " $a$ " and " $a+h$ ". Well, we are going to draw the secant line [see Figure 1] eh, [Anna traces it and repeat] And we are going to calculate the slope of that secant line ...it would be the tangent of this angle, but this angle is exactly the same as this one because it is between parallel lines, okay? Thales theorem, corresponding angles, okay? [...] it would be opposite leg divided by the adjacent leg, this is " $h$ ", therefore " $f(a+h)-f(a)) / h$ ", okay? This is what the textbook calls the average rate of change, eh, corresponding to point " $a$ " and an increase " $h$ "...

In this excerpt, Anna presents to her students the definition of the average rate of change (ARC, henceforth) in the interval "[a, $a+h]$ " through a generic example. According to Mason and Pimm (1984), a generic example "is an actual example, but one presented in such a way as to bring out its intended role as the carrier of the general" (p. 287). Anna uses a generic example because, although she uses a particular function, what she wants to show is its generality, i.e., how to calculate the ARC in three steps: first, the image of points "a" and "a+h" under $f$ (without calculating them explicitly), then their substraction and finally the quotient with the increase, " $h$ ". She repeats this way of proceeding when defining the derivative of a function at a point. Anna's repetitive way of acting is a characteristic of routines in the theory of commognition, so a routine (defining by exemplar) is inferred.

Regarding the visual mediators, Anna uses the graph of the function and the secant line, accompanied by symbolic visual mediators, to indicate the points and distances involved in the definition. In Figure 1, other symbolic visual mediators can be seen: the general equation of the secant line and the formula of the slope.

\section{RESULTS}

This section is organized in four subsections. In the first three, we classify various exploratory routines and visual mediators that appear when the teacher is introducing three different aspects of the derivative concept. The first subsection deals with the average rate of change, the second one with the derivative of a function at a point and the third one with the derivative function. Protocols and images of classroom episodes from the three sessions analyzed appear in each of these three subsections. The final subsection contains a summary of the results. 


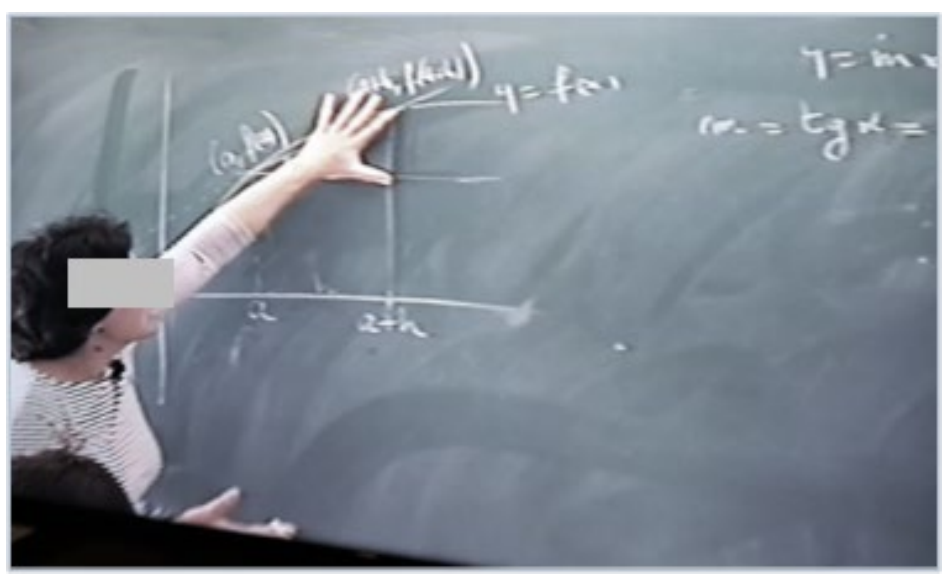

Figure 2. Explaining the ARC

\section{The Average Rate of Change (ARC)}

In the protocol that appears in the methodology section, Anna introduces a procedure to calculate the ARC in three steps, consisting of calculating the images of the points " $a+h$ " and " $h$ " under $f$, then their subtraction, and finally the quotient of it and the increase, "h". A construction routine is inferred from this: the teacher constructs the ARC definition by exemplar. It is a construction routine because it gives rise to an endorsed narrative: the definition itself, and it is a definition by exemplar because the teacher uses an example to introduce it; specifically, it uses a generic example of a graph of a function and some fixed points "a" and " $\mathrm{a}+\mathrm{h}$ ". The generic example used can be seen in Figure 1. We also observe a substantiation routine in the protocol, which can be classified as "verification of sufficient conditions" and even as a recall routine. Here Anna indicates to the students that the angles are equal because their respective sides are parallel and she reminds them of what Thales theorem states, which had been taught in a previous course. Although we have found many more recall routines in the transcripts analyzed, we will not discuss them in depth because all of these routines play a similar role.

The visual mediators used here are symbolic (symbols, formulas), graphic (function graph), and gestural. In Figure 2, a gestural visual mediator appears when Anna indicates with her hand the vertical distance involved in calculating the ARC. According to McNeill's (1992) classification, it is an iconic gesture because it visually refers to a concrete distance.

After introducing the ARC through a generic example, Anna focuses on concrete examples that appear in the textbook, with the motivation of clarifying the steps to follow in the computation of the ARC:

A: In the book, there are some exercises such as calculating the ARC at " $[1,2]$ ", then " $a$ " is 1, and " $a+h$ " is 2; or calculate the $A R C$ at point 1 with an increment of 0.5 , then it will be from 1 to 1.5 , okay? And that is the ARC because it would be " $f(a+h)-f$ (a)) / $h "$.

This shows that, at this level, generic examples may not be sufficient for students to reproduce the same procedures with concrete examples.

\section{Derivative of a Function at a Point f'(a)}

Anna introduces here the slope of the tangent line to a function at a point, which will be called later the derivative of a function at a point through naming. According to Viirman's (2014) classification, it is a construction routine, like the previous one, and it constructs the definition by exemplar using a generic example. As in the case of the ARC, this construction is based on the geometric meaning of the derivative of a function at a point: it is the slope of the tangent line at that point, i.e., the limit of the slopes of the secant lines. Anna also warns about a condition that is necessary to apply the definition (the when of the routine): that the function must be continuous (at this point), which from the mathematical point of view is a necessary condition for the derivative of the function to exist at this point. Unlike the previous one, this construction procedure cannot be carried out theoretically in a finite number of steps.

As in the previous subsection, after constructing the narrative that states the definition, Anna presents a concrete example. When Anna constructs a definition by using a generic example, we can identify that her discursive activity has the following structure: from the generic example, she constructs the narrative that states the definition and then gives a particular example.

As for visual mediators, Anna uses the graph of the function and the secant and tangent lines, accompanied by symbolic mediators, to indicate the points and distances in the graph. Figure 3 shows other symbolic visual mediators, the arrows from "a +h" to "a", which indicate successive approaches to the point "a". We can also observe a gestural visual mediator when Anna uses her arm and hand (which represent the secant lines) to illustrate how the slopes of the secant lines vary until they have the inclination of the tangent line. According to McNeill's (1992) classification, it is also an iconic gesture because the arm and hand can be visually identified with the lines they represent. When Anna represents with gestures the different secant lines, she is conveying the idea of the limit of these secant lines tending to the tangent line; therefore, it could be said that, as a whole, it is a metaphorical gesture (McNeill, 1992). 


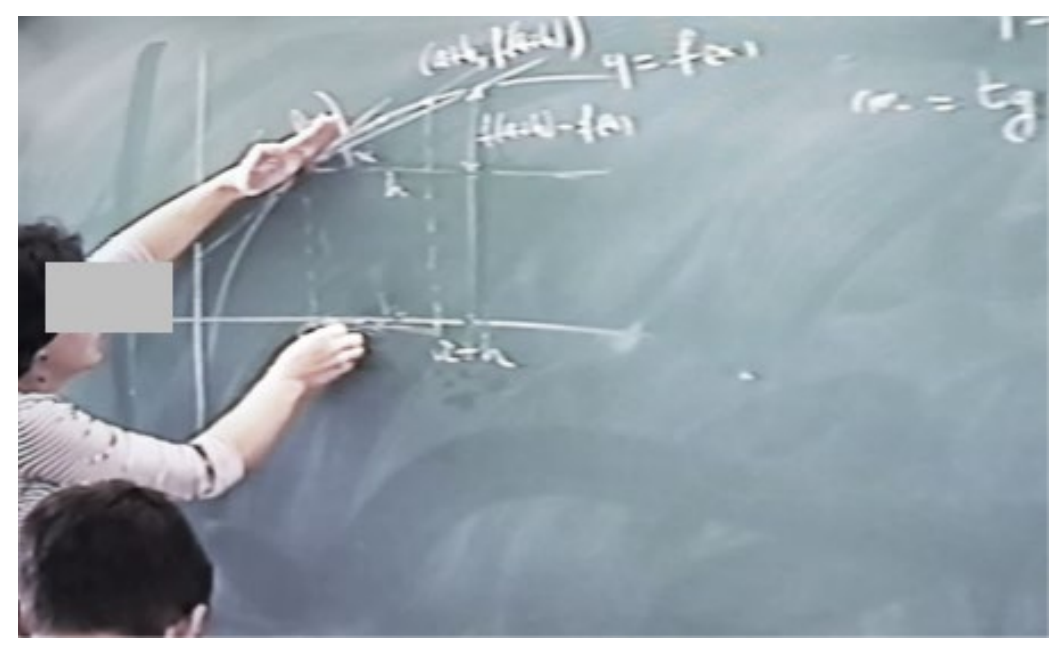

Figure 3. Gesture for the Variation of the Secant Lines

We show below the empirical evidence (protocol and image) for the construction of the slope of the tangent line.

A: Well, that straight line that is secant there, now what we are going to do is to tend, this " $h$ " we are going to make it smaller and smaller ... we are here, and we draw the secant line that goes through here and here, and we would calculate the ARC or the slope of the line, okay? We are getting closer and closer. If I get this point closer and closer to " $a$ " [see Figure 3], the line that is secant, where is it going? It ends to be the tangent line. We make the limit when " $h$ " tends to zero of " $f(a+h)-f(a)$ " divided by " $h$ " [Anna writes it at the same time] what is it going to be?... it is going to be the slope of the tangent line in "a" [Anna writes "slope of the tangent line in a, $f(a)$ "]. [...] Let's see for a moment, we have said that we are starting from continuous functions.

Besides, in the parts of the transcripts concerning this concept, we can observe construction routines, specifically the construction of definitions by naming, that is, the mathematical object has been introduced before, and the act of defining it simply consists of naming this new object (Viirman, 2014). For example, when the slope of the tangent line to the function at the point is designated as the "derivative of a function at a point".

A: Well, let's see, I haven't said everything yet, this is going to be the slope of the tangent line, but this is what is called the derivative of a function at a point " $a$ ", and is represented by "f" prime of " $a$ ".

Besides, another naming can be found when the slope of the tangent line (at a point) is named as the "slope of a curve" (at that point):

A: [...] but it is also called the slope of the curve because the curve there, at this point, coincides with the line at that point, and the slope is the slope of the tangent line.

The following construction routine usually appears after first presenting a definition. The generic example used to construct the definition of derivative already proved the existence of that mathematical object, which is a characteristic of the mathematical practice of defining (Martín-Molina et al., 2018). Still, the concrete example clarifies the steps that have to be followed to calculate the derivative at a point (the how of the routine), so we consider that this routine that constructs concrete examples has a didactic purpose. However, in the discourse of advanced mathematics, in which stipulative definitions are generally used, the example of the concept may appear for the first time in existence theorems, and in this case, this routine of construction of examples does not have a didactic purpose. We have identified numerous construction routines when presenting examples that serve to clarify the concepts introduced. Next, several examples of calculating the derivative of a function at a point using the limit definition are shown:

A: Come on, let's start; how much will "f" prime in 2 be worth? The definition please, limit of "f" of 2 plus " $h$ " minus "f" of 2 divided by " $h$ " [Anna points to the formula], when " $h$ " tends to zero, keep in mind that " $h$ " tending to zero, you know what it is, we have put " $h$ " here, but we could take it here [Anna points to the blackboard], " $h$ " tends to zero from both right and left, okay? Come on, how much is "f" of 2 plus " $h$ "?...

Student (S): 2, no, 2, no.

A: If "f" is three times " $x$ " squared, how much is "f" of scribble?

S: Three times scribble squared. 


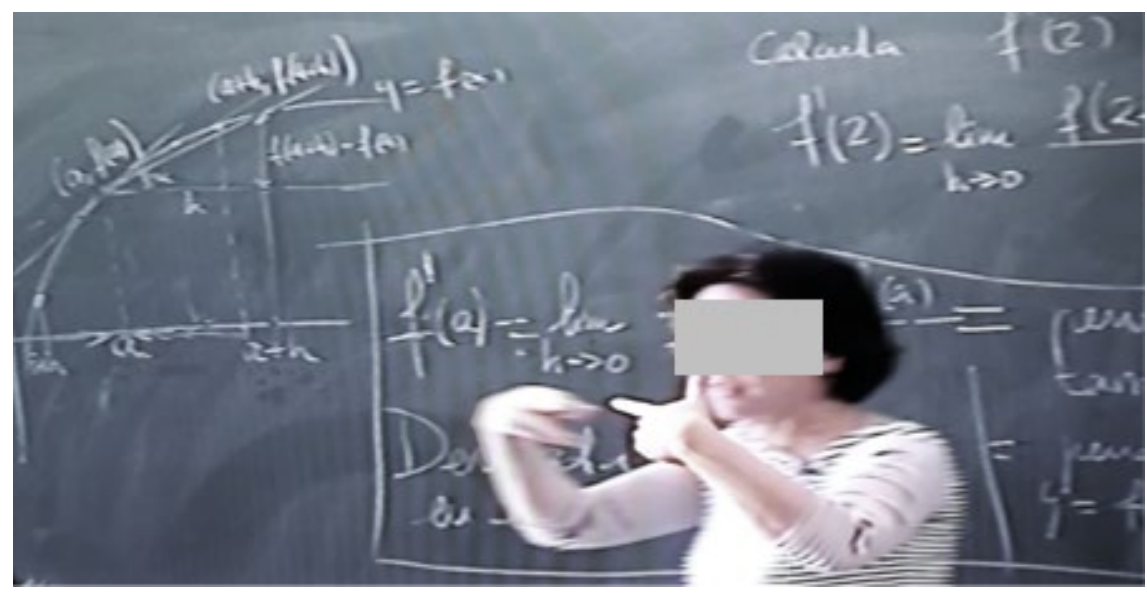

Figure 4. Approaching the Point from the Left and Right

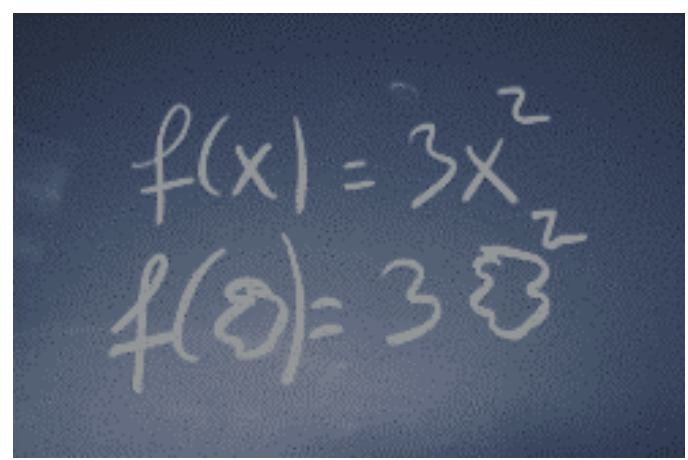

Figure 5. Finding the Image. The "Scribble"

Various visual mediators can be observed. In Figure 4, we have graphic and symbolic visual mediators, such as the graph of the function, the box used to highlight the definition of the derivative, and a gestural one, when Anna indicates that, when computing the limit, the approximation to the point is both from the right (higher values) and from the left (lower values). We consider it a metaphorical gesture (McNeill, 1992) because it represents the abstract idea of the limit process, where an informal realization of the limit based on movement is promoted (Güçler, 2013; Tall \& Vinner, 1981). In Figure 5, we can see another symbolic visual mediator, "the scribble", which Anna uses to represent the independent variable of the function and, in particular, each value it can take. Thus, the scribble is a metaphor that stands for a variable, an abstract entity, through something familiar and concrete for the student (a scribble) and as generic as a variable because it can take any form (Zandieh \& Knapp, 2006). Anna uses this visual mediator because she has observed that her students have difficulty calculating images of points, especially when they are not numbers, for example, the image of the point " $x+h$ ".

Substantiation routines can also be identified. We call the following one "checking necessary conditions". It consists of checking if some conditions are satisfied; otherwise, the associated narrative must be rejected. Specifically, a necessary condition for the derivative to exist at a point is that the limit of the difference quotient always gives rise to an indeterminate form $0 / 0$. Anna tells them that the numerator of the difference quotient, " $f(a+h)-f(a)$ ", must always tend to zero (because differentiability implies continuity). The denominator, " $h$ ", must also tend to zero.

Below, we present the protocol corresponding to this substantiation routine:

\section{A: What is the denominator going to be when we take the limit? What is it going to be?}

S: Zero.

A: And the numerator? When " $h$ " tends to zero and the function is continuous, how much will this be?

S: Zero.

A: That is, when we solve derivatives, we are going to have indeterminate forms zero divided by zero that we have to know how to solve, eh...

The following protocol is another example of the same routine ("checking necessary conditions"):

A: Therefore, that definition that we have put there is exactly like that, and the " $a$ " is worth minus 2, and the " $h$ " is still " $h$ ", eh, we make the limit when " $h$ " tends to 0 , so here the variable is " $h$ ", the variable here is always " $h$ " [she points it out], always, 


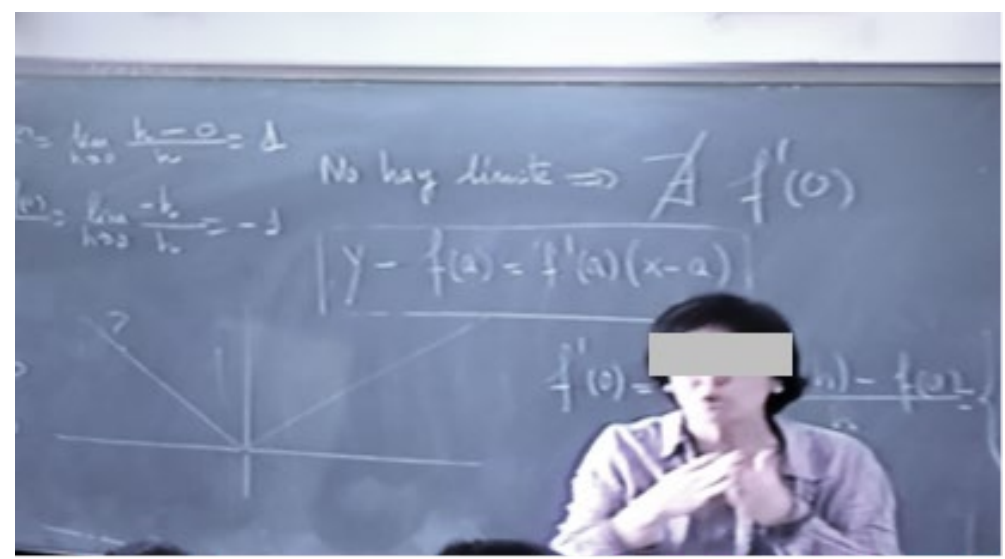

Figure 6. Gesture Indicating a Peak

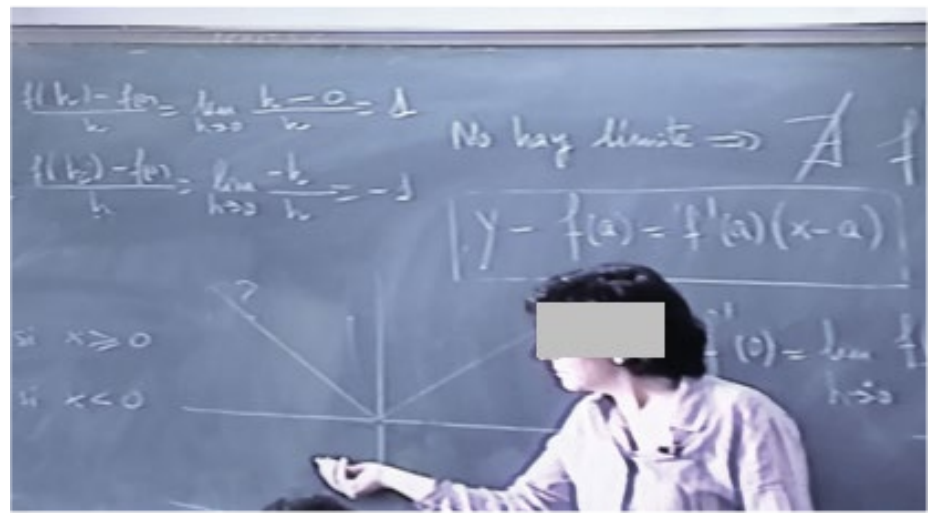

Figure 7. "Half Tangent Line" of the Absolute Value at the Origin

always it is going to be an indeterminate form $0 / 0$ eh, as long as we are doing well, that is what you have to think now, if I get an indeterminate form 0/0, then I am going on the right track, eh, I can be wrong but if I get something other than 0/0...

The following substantiation routine is of the type "claim contradiction", consisting of using examples to contradict statements (Viirman, 2014). The following excerpt shows a routine that contradicts the statement "all continuous functions are differentiable", using the absolute value function at the origin of the coordinate system as a generic example:
A: [...] This function is continuous here, but it breaks [Anna makes a gesture], what happens here? If we want to calculate the tangent line, what happens at that point?
S: That you would have different tangents.
A: That you would have a tangent line over here [Anna makes the gesture on the graph] and another tangent line over here, but not a single tangent line, it seems that there are about two half lines... two half tangent lines, one on one side and one on the other, that is to say, the function is continuous, but here at this point there is no derivative, therefore, the tangent line cannot be calculated, okay?

In the previous episode, we can also find two iconic gestural visual mediators, shown in Figures 6 and 7 . They are iconic because they can be visually identified with their concrete referents: a peak (Figure 6) and the "half tangent lines" (Figure 7).

\section{Derivative Function $f^{\prime}(x)$}

Next, we can find another construction routine, when Anna constructs the definition of the derivative function. It is constructed through an algebraization process, called "genericization" by Panero et al. (2006), because it merely consists of substituting the point "a" ("a" represents a generic example of a concrete number) by the variable " $x$ " in the definition of the derivative of a function at a point. As Lakoff and Núñez (2000) mention, this is the "fundamental metonymy of algebra". This fundamental metonymy may have appeared in previous courses, at least when the concept of function was studied. The following protocol reflects this routine:

\footnotetext{
A: The derivative function ... [Anna writes " $f$ ' $(x)$ "], how will it be?

S: ... it will be "f" of " $x$ " plus " $h$ " ...
}

A: How do we start? 


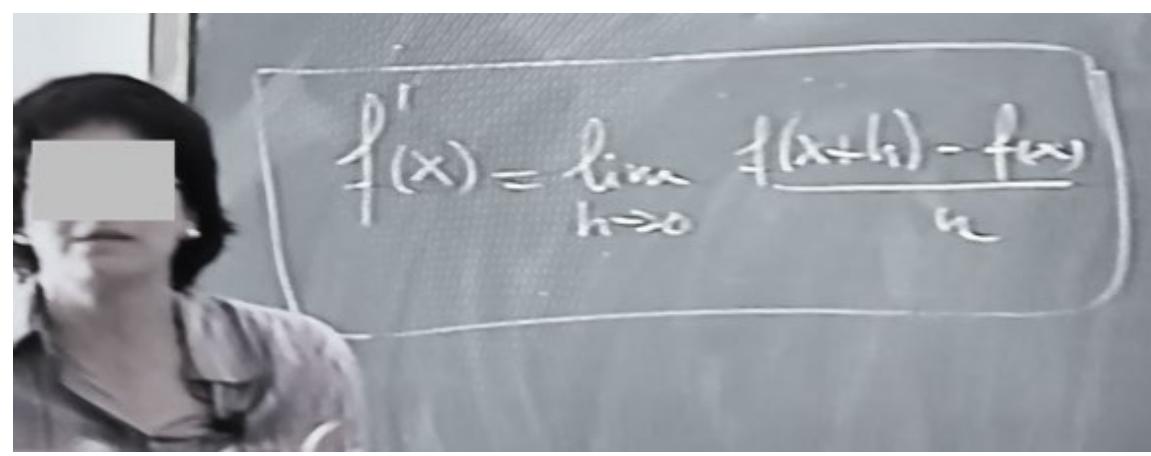

Figure 8. The Formula of the Derivative Function

\section{S: [All at once] limit...}

A: Limit...

S: ... when " $h$ " tends to zero of "f" of " $x+h$ " minus " $f$ " of " $x$ " divided by " $h$ " [Anna writes it on the board inside a box]

A: This will be..., instead of in " $a$ " we will do it in a generic " $x$ ", eh, and it will be the derivative function of "f", okay? It will have a domain, that will be the set of points in which the derivative of " $f$ " exists, eh, okay? And so, the derivative function will be calculated like this.

In the image corresponding to the previous protocol, we find the general formula for computing the derivative function, a symbolic visual mediator (see Figure 8).

The following routine is also a substantiation routine, of "definition verification", and consists of checking the result using the geometric definition. Specifically, when the function to be derived is a line, the result obtained using the limit of the difference quotient must coincide with the slope of the line, since the tangent line is the line itself. The slope of the line can be obtained directly from the equation of the line, for example, from the explicit equation ( $y=m x+n)$, where the coefficient of $x$, " $m$ ", is the slope of the line. Thus, the new knowledge built is consistent with the previous one. This is what Shulman (1986) refers to as "vertical curriculum". Here we present the protocol that we refer to above:

A: This is the function eh, if they give you [Anna gives another example and draws it] a line and they tell you: calculate the derivative of " $y$ " equal to " $3 x+1$ " at point 1 , then it will be 3 , and calculate the derivative at 0 , again it will be 3, because it is a line eh and the slope of this line is 3, and what I am calculating when I find a derivative is the slope of the tangent line itself, then it is always, always, always 3.

Finally, we have inferred a routine of substantiation algorithms. These routines use algorithms to justify claims (Viirman, 2014). Specifically, in this episode, Anna proposes to use the derivative rule to check if the derivative function has been calculated correctly as the limit of the difference quotient. Anna refers here to the "horizontal curriculum" (Shulman, 1986) since the derivative rule for the power function has been studied previously in a physics course to calculate speeds and accelerations. Specifically, she refers to the derivative of the function " $x^{n}$ ", which is equal to " $n x^{n-1}$ ".

A: They tell me, calculate the derivative of "f" of " $x$ " equal to " $x$ " squared [Anna writes it], shh, please ...you already know, but you can do it now by calculating the limit, you know the derivative of this function, it is 2 times " $x$ ". And the derivative of " $x$ " cube?

S: 3 times " $x$ " squared,

A: 3 times " $x$ " squared, you know, you've already done it in physics, well, let's see, shh, well, now you're going to do it and calculate the limits because you know how to do it.

\section{Summary of the Results}

We have found and classified exploratory routines of construction, substantiation, and recall. We present below a summary of the types that were found:

- The construction routines identified are definition construction by exemplar (through generic examples), naming, and construction of examples.

- The substantiation routines identified are definition verification, substantiation algorithms, verification of sufficient conditions, and checking necessary conditions.

- Recall routines are numerous in the discursive activity of Anna but all of them fulfill the same function and are not directly related to the derivative concept. This is the reason we have only shown one example and focused on the other two subtypes of exploratory routines. 
Regarding the visual mediators, we have found several graphic, symbolic, and gestural ones. Although gestures of the four types (iconic, metaphorical, deixis, and beat) appeared, we have centered on the first two types since deictic and beat gestures are not directly linked to mathematical content (Farsani et al., 2020).

\section{CONCLUSIONS AND DISCUSSION}

In this work, we have identified routines and visual mediators in an upper secondary school teacher's discursive activity when she introduces the derivative concept. We have found various types of visual mediators, such as graphic, symbolic, and gestural. We have also identified exploratory routines of construction, substantiation, and recall (Sfard, 2008).

Regarding construction routines, we have identified construction of definitions by exemplar and naming (Viirman, 2014). While in previous educational stages, definitions are often descriptive or with concrete examples (Kobiela \& Lehrer, 2015; Tall, 1991; Vinner, 1991; Zaslavsky \& Shir), in our case, the definitions by exemplar that were found used generic examples. Kaminski et al. (2008) state that the use of generic examples instead of concrete ones may be advantageous because

instantiating an abstract concept in a concrete, contextualized manner appears to constrain that knowledge and to hinder the ability to recognize the same concept elsewhere; this, in turn, obstructs knowledge transfer. At the same time, learning a generic instantiation allows for transfer, which suggests that such an instantiation could result in a portable knowledge representation. (p. 455)

We think that this capacity of knowledge transfer is crucial for the first years of university and should be promoted, but also that generic examples should be used cautiously, because they may fail their purpose, since, as Mason and Pimm (1984) state, it is almost impossible to tell whether someone is noticing or ignoring what you intend by giving a generic example. In our case, the use of this type of examples implies that, for these students, a concrete example is also necessary so that they can give full meaning to the definition and so that they can see not only the general but also the particular (the specific numerical values).

Unlike the work of Viirman (2014), no stipulative definitions have been found in our study. This kind of definition can be considered more typical of university levels (Edwards \& Ward, 2008), in which teaching very frequently follows the DTP (definitiontheorem-proof) scheme (Weber, 2004). In this format

the construction of the formal definition and consequent creation of a technical term is thus purposeful and creative, aiming not simply to describe or capture a pre-existing concept but to shape that concept in a way that lends itself to particular purposes. Of course, this definition may subsequently be used to generate deductive sequences leading to the discovery of further theorems. (Morgan, 2005, p. 862)

Thus, while definitions at school are usually descriptive, closer to the students' experience, at university, they are typically introduced for constructive and deductive purposes, for example, to create and prove theorems (Morgan, 2005, p. 862). In this case, students frequently do not have any image of the concept that is introduced through the definition, contrary to what happens at school (Edwards \& Ward, 2008).

Consequently, we consider that the routines that construct definitions by exemplar with generic examples can help students in their transition to academic mathematics since they are halfway between definitions by exemplar with concrete examples and stipulative definitions, in the sense of the degree of abstraction.

We have also found exploratory routines that construct definitions by naming. For example, they appear when the slope of a curve (at a point) is defined. On the other hand, we have found an instance of definition that would not consist simply of giving a name to an object but in going one step further by changing the numerical set, through generalizations, or extending the scope of definitions. For example, in solving second-degree equations in $\mathbb{R}$ (real numbers), if we consider the solution of the same equations in $\mathbb{C}$ (complex numbers), the concept of solving an equation is the same, but in $\mathbb{C}$ the equation always has a solution. In particular, in our work, there is a definition that consists of using the fundamental metonymy of algebra and then naming the object. Thus, the derivative function is defined based on an object constructed before, the derivative of a function at a point, by changing the generic point for the variable and by then giving it the name of derivative function. According to the curriculum, the fundamental metonymy of algebra, established in the passage from arithmetic to algebra, can be used before, for example, when the concept of function is considered (in the Spanish curriculum, in middle secondary education, 12-15 years). However, we think that more construction routines of the last type may appear in the transition to university than in previous courses due to its greater complexity. In fact, Rasmussen et al. (2005) state that "the practice of defining can function both as an organizing activity [...] and as a means for generalizing, formalizing or creating a new mathematical reality" (p. 66). Then we think this is a resource that students can use to reason about generality (for example, trying to generalize definitions) rather than merely about the particular.

Regarding substantiation routines, we have found some types that complement the work by Viirman (2014). One of the new types of routines found in our study is "verification of sufficient conditions". This type of routine is useful to justify why a narrative is endorsed. In our case, it appears when Anna affirms that the rays that form two angles are parallel, and then she deduces that these angles are equal. These conditions are not necessary in the sense that one angle can be equivalent to another, but the sides of the first may not be parallel to those of the second. Another new kind of routine identified in our work is "checking necessary conditions", and in this case, the rejection of a narrative is justified; for example, if the corresponding limit does not create an indeterminate form $0 / 0$, then the narrative "the derivative of the function at that point exists" can be rejected. Similarly, checking 
that these necessary conditions are met is not useful to endorse the previous narrative since it may occur that, when solving the indeterminate form, the final result is equal to plus or minus infinity, so the condition is not sufficient. As in the work by Viirman (2014), which is set in the first year of university, we have also found "definition verification" and "substantiation algorithms" routines. The "definition verification" routine found consists of combining two definitions, one of them already worked on in previous courses: the geometric definition of derivative as the slope of the tangent line and the geometrical-analytic definition of the slope of a line, as "the coefficient of the independent variable in the explicit equation of the line"; they are used here to check if the calculation of the derivative in the case of a line is correct. This combination of definitions gives students evidence that different valid definitions may exist for the same concept, and each one can be useful depending on the problem to solve.

The substantiation routines mentioned are not exclusive to the transition to advanced mathematics. In primary education, we can already find some of them; a "substantiation algorithm" routine that sometimes appears on this stage is the realization of the division test (the dividend must be equal to the divisor times the quotient plus the remainder) to check in turn that another algorithm, that of the division, is carried out without errors. At this level, we can also find "definition verification" routines, for example, when a student justifies that a particular instance of a polygon is a triangle. However, we think that these and other types of substantiation routines (especially those in terms of necessary and sufficient conditions) are not usual at school, because students often do not understand the role that these routines play in the problem-solving process (Knuth, 2002; Nardi et al., 2014; Sfard, 2008; Tall, 1991; Thoma \& Nardi, 2018). Then, we think that substantiation routines begin to have more importance in the transition to university and help students understand more deeply the structure and rigor of mathematics.

We have also found several recall routines and all of them serve the same function. According to Sfard (2008), recall type routines are useful "because explorations build on previously endorsed narratives, remembering a certain amount of those former narratives is important for one's discursive fluency” (p. 234). Regarding this type of routines, Viirman (2014) points out that they are infrequent in the lecturers' discursive activity at university since lectures are given in this educational stage with a highly prepared speech and without room for improvisation. We consider that the recall routines that were found have a didactic character. In fact, Viirman (2015), in his work about the teacher's pedagogical discourse, identifies didactic routines of "explanation", where some of its subtypes are closely related to recall routines, such as "known mathematical facts" and "summary and repetition". These routines cannot be considered exclusively characteristic of the secondary-tertiary transition; they are also very frequent in primary education and the first years of secondary education because they are essential from the didactic point of view due to the cumulative and interconnected nature of mathematics. Thus, these routines help the student to organize the contents, that is, they do not only help to remember a particular concept but also show how various concepts are related to each other.

As for visual mediators, we have found various types, such as graphic, symbolic, and gestural. Symbols represent the processobject duality of mathematical concepts, so they reduce cognitive load. It is expected that their use in upper secondary education will be greater than in middle school since they support the transition to advanced mathematical thinking (Gray \& Tall, 1994; Sfard, 1991). Several gestural visual mediators have also been identified, and the iconic ones, which visually resemble what they represent, are the most used, hence we consider them characteristic of school discourse. Gestures that refer to abstract entities, which are named "metaphorical" by McNeill (1992), are quite frequent in mathematics (Edwards, 2009), but we have found few examples of them in the discourse analyzed, for instance, the one in Figure 4 that reflects a dynamic idea of the concept of limit (Güçler, 2013; Tall \& Vinner 1981). We consider that metaphorical gestures representing infinite processes (for example, limits) are more typical of the transition stage than previous stages due to the more abstract nature of these concepts.

As Artigue (1991) and Cornu (1991) point out, due to the difficulty of the formalizations in analysis, the stipulative epsilon-delta definition of the derivative, for example, should not be introduced at upper secondary education; they argue that students in this stage still have problems in understanding the quantifiers since in mathematics some of them have a non-intuitive meaning. Moreover, analyzing subsequent lessons, we have noticed that Anna ends up resorting to the "algebraization" of analysis: she manipulates formulas instead of functions, gives more importance to the computation of derivatives than to the meaning of the concept, and pays little attention to the graphical mode. In this respect, Alcock and Simpson (2002) state that "certain reasoning strategies are inadequate when applied to university mathematics, although they might be efficient and sufficient in the kind of reasoning with specific objects required by school mathematics" (p. 33). Therefore, the only use of these strategies in upper secondary education may create difficulties for students in their first years of university. Finally, we agree with Niss (2003) that the coherence of mathematics as a subject at all educational levels is necessary to guarantee success in the transition to university and should be promoted in teachers' discursive activities and practices.

A limitation of this work is that we studied the mathematical discursive activity of only one teacher when she taught a specific concept in a specific course. In the future, we intend to examine the discursive activity of more teachers when teaching this and other mathematical concepts. Finally, we would like to complement this study of the mathematical discourse by analyzing the teacher's pedagogical discursive activity.

Author contributions: All authors have sufficiently contributed to the study, and agreed with the results and conclusions.

Funding: The first author was partially supported by the "VI Plan Propio de Investigación y Transferencia" of the Universidad de Sevilla (Spain). Both authors belong to the Research Group in Mathematics Education FQM-226 of the Junta de Andalucía (Spain).

Declaration of interest: No conflict of interest is declared by authors.

\section{REFERENCES}

Alcock, L., \& Simpson, A. (2002). Definitions: Dealing with categories mathematically. For the learning of mathematics, 22(2), $28-34$. 
Alibali, M. W., \& Nathan, M. J. (2012). Embodiment in mathematics teaching and learning: Evidence from learners' and teachers' gestures. Journal of the Learning Sciences, 21(2), 247-286. https://doi.org/10.1080/10508406.2011.611446

Artigue, M. (1991). Analysis. In D. Tall (Ed.), Advanced mathematical thinking (pp. 167-198). Kluwer.

Badillo, E., Azcarate, C., \& Font, V. (2011). Análisis de los niveles de comprensión de los objetos $f(a)$ y $f(x)$ en profesores de matemáticas [Analysis of mathematics teachers' level of understanding of the objects $f^{\prime}(a)$ and $f^{\prime}(x)$ ]. Enseñanza de las Ciencias, 29(2), 191-206. https://doi.org/10.5565/rev/ec/v29n2.546

Bayda, N. I., \& Sutliff, G. (2020). Comparing extracted and stipulated definitions in Algebra 1 textbooks and Khan Academy. International Electronic Journal of Mathematics Education, 15(2), 1-8. https://doi.org/10.29333/iejme/7601

Behr, M., Lesh, R., Post, T., \& Silver, E. (1983). Rational number concepts. In R. Lesh (Ed.), Acquisition of mathematical concepts and processes (pp. 91-126). New Academic Press.

Biza, I., Giraldo, V., Hochmuth, R., Khakbaz, A. S., \& Rasmussen, C. (2016). Research on teaching and learning mathematics at the tertiary level: State-of-the-art and looking ahead. Springer. https://doi.org/10.1007/978-3-319-41814-8

Borji, V., \& Sánchez, A. (2019). An exploratory analysis of the representations of functions in the university entrance exam in Spain and Iran. Eurasia Journal of Mathematics, Science and Technology Education, 15(8), em1727. https://doi.org/10.29333/ejmste/106258

Borji, V., Font, V., Alamolhodaei, H., \& Sánchez, A. (2018). Application of the complementarities of two theories, APOS and OSA, for the analysis of the university students' understanding on the graph of the function and its derivative. Eurasia Journal of Mathematics, Science and Technology Education, 14(6), 2301-2315. https://doi.org/10.29333/ejmste/89514

Burn, R. (2002). The genesis of mathematical structures. In P. Kahn \& J. Kyle (Eds.), Effective learning and teaching in mathematics and its applications (pp. 20-33). Kogan Page.

Clark, M., \& Lovric, M. (2009). Understanding secondary-tertiary transition in mathematics. International Journal of Mathematical Education in Science and Technology, 40(6), 755-776. https://doi.org/10.1080/00207390902912878

Cornu, B. (1991). Limits. In D. Tall (Ed.), Advanced mathematical thinking (pp. 153-166). Kluwer.

Corriveau, C., \& Bednarz, N. (2016). The secondary-tertiary transition viewed as a change in mathematical cultures: An exploration concerning symbolism and its use. Educational Studies in Mathematics, 95(1), 1-19 (2017). https://doi.org/10.1007/s10649-0169738-z

Dahl, B. (2017). First-year non-STEM majors' use of definitions to solve calculus tasks: Benefits of using concept image over concept definition? International Journal of Science and Mathematics Education, 15(7), 1303-1322. https://doi.org/10.1007/s10763-0169751-9

De Guzman, M., Hodgson, B., Robert, A., \& Villani, V. (1998). Difficulties in the passage from secondary to tertiary education. In G. Fischer \& U. Rehmann (Eds.), Proceedings of the International Congress of Mathematicians (Vol III, pp. 747-762). Fakultät für Mathematik, Universität Bielefeld.

De Villiers, M. (1998). To teach definitions in geometry or teach to define? In A. Olivier \& K. Newstead (Eds.), Proceedings of the 22nd conference of the International Group for the Psychology of Mathematics Education (Vol. 2, pp. 248-255). University of Stellenbosch.

Dreher, A., Lindmeier, A., Heinze, A., \& Niemand, C. (2018). What kind of content knowledge do secondary mathematics teachers need? Journal für Mathematik-Didaktik, 39(2), 319-341. https://doi.org/10.1007/s13138-018-0127-2

Edwards, B., \& Ward, M. (2008). The role of mathematical definitions in mathematics and in undergraduate mathematics courses. In M. Carlson \& C. Rasmussen (Eds.), Making the connection: Research and teaching in undergraduate mathematics (pp. 221230). Mathematical Association of America.

Edwards, L. D. (2009). Gestures and conceptual integration in mathematical talk. Educational Studies in Mathematics, 70(2), 127141. https://doi.org/10.1007/s10649-008-9124-6

Emre-Akdoğan, E., Güçler, B., \& Argün, Z. (2018). The development of two high school students' discourses on geometric translation in relation to the teacher's discourse in the classroom. Eurasia Journal of Mathematics, Science and Technology Education, 14(5), 1605-1619. https://doi.org/10.29333/ejmste/84885

Farsani, D., Breda, A., \& Sala, G. (2020). ¿Cómo los gestos de los maestros afectan a la atención visual de las estudiantes durante el discurso matemático? [How do teachers' gestures affect students' visual attention during mathematical discourse?]. REDIMAT - Journal of Research in Mathematics Education, 9(3), 220-242. https://doi.org/10.17583/redimat.2020.5185

Freudenthal, H. (1973). Mathematics as an educational task. Reidel.

Fuentealba, C., Badillo, E., \& Sánchez-Matamoros, G. (2019). Identificación y caracterización de los subniveles de desarrollo del esquema de derivada [Identification and characterization of the development sublevels of the derivative schema]. Enseñanza de las Ciencias, 37(2), 63-84. https://doi.org/10.5565/rev/ensciencias.2518

Fuentealba, C., Badillo, E., Sánchez-Matamoros, G., \& Cárcamo, A. (2019). The understanding of the derivative concept in higher education. Eurasia Journal of Mathematics, Science and Technology Education, $15(2)$, em1662. https://doi.org/10.29333/ejmste/100640 
García, M., Gavilán, J. M., \& Llinares, S. (2012). Perspectiva de la práctica del profesor de matemáticas de secundaria sobre la enseñanza de la derivada. Relaciones entre la práctica y la perspectiva del profesor [Perspective of the secondary mathematics teacher's practice on the teaching of the derivative. Relations between the teacher's perspective and practice]. Enseñanza de las Ciencias, 30(3), 219-235. https://doi.org/10.5565/rev/ec/v30n3.684

Gray, E. M., \& Tall, D. O. (1994). Duality, ambiguity and flexibility: A "proceptual” view of simple arithmetic. Journal for Research in Mathematics Education, 25(2), 116-140. https://doi.org/10.2307/749505

Güçler, B. (2013). Examining the discourse on the limit concept in a beginning-level calculus classroom. Educational Studies in Mathematics, 82(3), 439-453. https://doi.org/10.1007/s10649-012-9438-2

Gueudet, G. (2008). Investigating the secondary-tertiary transition. Educational Studies in Mathematics, 67(3), $237-254$. https://doi.org/10.1007/s10649-007-9100-6

Kaminski, J. A., Sloutsky, V. M., \& Heckler, A. F. (2008). The advantage of abstract examples in learning math. Science, 320(5875), 454-455. https://doi.org/10.1126/science.1154659

Knuth, E. J. (2002). Secondary school mathematics teachers' conceptions of proof. Journal for Research in Mathematics Education, 33(5), 379-405. https://doi.org/10.2307/4149959

Kobiela, M., \& Lehrer, R. (2015). The codevelopment of mathematical concepts and the practice of defining. Journal for Research in Mathematics Education, 46(4), 423-454. https://doi.org/10.5951/jresematheduc.46.4.0423

Lakatos, I. (1976). Proofs and refutations. Cambridge University Press.

Lakoff, G., \& Núñez, R. E. (2000). Where mathematics comes from: How the embodied mind brings mathematics into being. Basic Books.

Landau, S. I. (2001). Dictionaries: The art and craft of lexicography (2nd ed.). Cambridge University Press.

Lavie, I., Steiner, A., \& Sfard, A. (2019). Routines we live by: From ritual to exploration. Educational Studies in Mathematics, 101(2), 153-176. https://doi.org/10.1007/s10649-018-9817-4

Lerman, S. (2001). Cultural, discursive psychology: A sociocultural approach to studying the teaching and learning of mathematics. Educational Studies in Mathematics, 46(1-3), 87-113. https://doi.org/10.1023/A:1014031004832

Martín-Molina, V., González-Regaña, A. J., \& Gavilán-Izquierdo, J. M. (2018) Researching how professional mathematicians construct new mathematical definitions: A case study. International Journal of Mathematical Education in Science and Technology, 49(7), 1069-1082. https://doi.org/10.1080/0020739X.2018.1426795

Martín-Molina, V., González-Regaña, A. J., Toscano, R., \& Gavilán-Izquierdo, J. M. (2020). Differences between how undergraduate students define geometric solids and what their lecturers expect from them through the lens of the theory of commognition. Eurasia Journal of Mathematics, Science and Technology Education, 16(12), em1917. https://doi.org/10.29333/ejmste/9159

Mason, J., \& Pimm, D. (1984). Generic examples: Seeing the general in the particular. Educational Studies in Mathematics, 15(3), 277-289. https://doi.org/10.1007/BF00312078

McNeill, D. (1992). Hand and mind: What gestures reveal about thought. University of Chicago Press.

Morgan, C. (2005). Word, definitions and concepts in discourses of mathematics, teaching and learning. Language and Education, 19(2), 102-116. https://doi.org/10.1080/09500780508668666

Nardi, E., Ryve, A., Stadler, E., \& Viirman, O. (2014). Commognitive analyses of the learning and teaching of mathematics at university level: The case of discursive shifts in the study of Calculus. Research in Mathematics Education, 16(2), 182-198. https://doi.org/10.1080/14794802.2014.918338

Niss, M. (2003). Mathematical competencies and the learning of mathematics: The Danish KOM project. In A. Gagatsis \& S. Papastavridis (Eds.), Proceedings of the 3rd Mediterranean Conference on Mathematics Education (pp. 115-124). Hellenic MS.

Panero, M., Arzarello, F., \& Sabena, C. (2016). The mathematical work with the derivative of a function: Teachers' practices with the idea of "generic". Bolema, Rio Claro (SP), 54(30), 265-286. https://doi.org/10.1590/1980-4415v30n54a13

Park, J. (2011). Calculus instructors' and students' discourses on the derivative (Unpublished doctoral dissertation). Michigan State University, Michigan.

Park, J. (2015). Is the derivative a function? If so, how do we teach it? Educational Studies in Mathematics, 90(2), $233-250$. https://doi.org/10.1007/s10649-015-9630-2

Pinto-Rojas, I. E., \& Parraguez, M. (2017). Articulators for thinking modes of the derivative from a local perspective. International Electronic Journal of Mathematics Education, 12(3), 873-898.

Planas, N., \& Civil, M. (2010). Discourse processes in critical mathematics education. In H. Alrø, O. Ravn, \& P. Valero (Eds.), Critical mathematics education: Past, present and future (pp. 145-160). Sense Publishers.

Rach, S., \& Heinze, A. (2017). The transition from school to university in mathematics: Which influence do school-related variables have? International Journal of Science and Mathematics Education, 15(7), 1343-1363. https://doi.org/10.1007/s10763-016-97448

Rach, S., \& Ufer, S. (2020). Which prior mathematical knowledge is necessary for study success in the university study entrance phase? Results on a new model of knowledge levels based on a reanalysis of data from existing studies. International Journal of Research in Undergraduate Mathematics Education, 6(3), 375-403. https://doi.org/10.1007/s40753-020-00112-x 
Rasmussen, C., Zandieh, M, King, K., \& Teppo, A. (2005). Advancing mathematical activity: A practice-oriented view of advanced mathematical thinking. Mathematical Thinking and Learning, 7(1), 51-73. https://doi.org/10.1207/s15327833mtl0701_4

Rooch, A., Junker, P., Härterich, J., \& Hackl, K. (2016). Linking mathematics with engineering applications at an early stage Implementation, experimental set-up and evaluation of a pilot project. European Journal of Engineering Education, 41(2), 172191. https://doi.org/10.1080/03043797.2015.1056095

Schüler-Meyer, A. (2018). Defining as discursive practice in transition - Upper secondary students reinvent the formal definition of convergent sequences. In V. Durand-Guerrier, R. Hochmuth, S. Goodchild, \& N. M. Hogstad (Eds.), Proceedings of INDRUM 2018 (pp. 255-264). University of Agder.

Sfard, A. (1991). On the dual nature of mathematical conceptions: Reflections on processes and objects as different sides of the same coin. Educational Studies in Mathematics, 22(1), 1-36. https://doi.org/10.1007/BF00302715

Sfard, A. (2008). Thinking as communicating human development, the growth of discourses, and mathematizing. Cambridge University Press.

Sfard, A. (2009). What's all the fuss about gestures? A commentary. Educational Studies in Mathematics, 70(2), $191-200$. https://doi.org/10.1007/s10649-008-9161-1

Sfard, A. (2014). University mathematics as a discourse - Why, how, and what for? Research in Mathematics Education, 16(2), 199203. https://doi.org/10.1080/14794802.2014.918339

Sfard, A. (2007). When the rules of discourse change, but nobody tells you: Making sense of mathematics learning from a commognitive standpoint. Journal of the Learning Sciences, 16(4), 565-613. https://doi.org/10.1080/10508400701525253

Shulman, L. S. (1986). Those who understand: Knowledge growth in teaching. Educational Researcher, 15(2), 4-14. https://doi.org/10.3102/0013189X015002004

Tabach, M., \& Nachlieli, T. (2016). Communicational perspectives on learning and teaching mathematics: Prologue. Educational Studies in Mathematics, 91(3), 299-306. https://doi.org/10.1007/s10649-015-9638-7

Tall, D. O. (1991). The psychology of advanced mathematical thinking. In D. O. Tall (Ed.), Advanced mathematical thinking (pp. 124). Kluwer.

Tall, D., \& Vinner, S. (1981). Concept image and concept definition in mathematics with particular reference to limits and continuity. Educational Studies in Mathematics, 12(2), 151-169. https://doi.org/10.1007/BF00305619

Tasara, I. (2018). Commognitive analysis of a teacher's mathematical discourse on the derivative. In F. Curtis (Ed.), BSRLM Proceedings, 2017: British Society for Research into Learning Mathematics Conference. British Society for Research into Learning Mathematics.

Thoma, A., \& Nardi, E. (2018). Transition from school to university mathematics: Manifestations of unresolved commognitive conflict in first year students' examination scripts. International Journal of Research in Undergraduate Mathematics Education, 4(1), 161-180. https://doi.org/10.1007/s40753-017-0064-3

Thomas, M. O. J., de Freitas Druck, I., Huillet, D., Ju, M. K., Nardi, E., Rasmussen, C., \& Xie, J. (2015). Key mathematical concepts in the transition from secondary school to university. In S. J. Cho (Ed.), The proceedings of the 12th international congress on mathematical education (pp. 265-284). Springer.

Viirman, O. (2014). The functions of function discourse - University mathematics teaching from a commognitive standpoint. International Journal of Mathematical Education in Science and Technology, 45(4), 512-527. https://doi.org/10.1080/0020739x.2013.855328

Viirman, O. (2015). Explanation, motivation and question posing routines in university mathematics teachers' pedagogical discourse: A commognitive analysis. International Journal of Mathematical Education in Science and Technology, 46(8), 11651181. https://doi.org/10.1080/0020739X.2015.1034206

Vinner, S. (1991). The role of definitions in the teaching and learning of mathematics. In D. Tall (Ed.), Advanced mathematical thinking (pp. 65-81). Kluwer.

Weber, K. (2004). Traditional instruction in advanced mathematics courses: A case study of one professor's lectures and proofs in an introductory real analysis course. Journal of Mathematical Behavior, 23(2), $115-133$. https://doi.org/10.1016/j.jmathb.2004.03.001

Yin, R. K. (2003). Case study research: Design and methods (3rd ed. ). Sage Publications.

Zandieh, M. J. (2000). A theoretical framework for analyzing student understanding of the concept of derivative. CBMS Issues in Mathematics Education, 8, 103-122.

Zandieh, M., \& Knapp, J. (2006). Exploring the role of metonymy in mathematical understanding and reasoning: The concept of derivative as an example. Journal of Mathematical Behavior, 25(1), 1-17. https://doi.org/10.1016/j.jmathb.2005.11.002

Zaslavsky, O., \& Shir, K. (2005). Students' conceptions of a mathematical definition. Journal for Research in Mathematics Education, 36(4), 317-346. 